\title{
Pioglitazone Identifies a New Target for Aneurysm Treatment: Role of Egr 1 in an Experimental Murine Model of Aortic Aneurysm
}

\author{
Nicoletta Charolidi Grisha Pirianov Evelyn Torsney Stuart Pearce Ken Laing \\ Axel Nohturfft Gillian W. Cockerill \\ Institute of Cardiovascular and Cell Sciences, St. George's, University of London, London, UK
}

\section{Key Words}

Aneurysm · Angiotensin II · Macrophage · Pioglitazone ·

Polycystic kidney disease

\begin{abstract}
Peroxisome proliferator-activated receptor $\gamma$ agonists have been shown to inhibit angiotensin II (Angll)-induced experimental abdominal aortic aneurysms. Macrophage infiltration to the vascular wall is an early event in this pathology, and therefore we explored the effects of the peroxisome proliferator-activated receptor $\gamma$ agonist pioglitazone on Angll-treated macrophages. Using microarray-based expression profiling of phorbol ester-stimulated THP-1 cells, we found that a number of aneurysm-related gene changes effected by Angll were modulated following the addition of pioglitazone. Among those genes, polycystic kidney disease 1 (PKD1) was significantly up-regulated (multiple testing corrected $p<0.05$ ). The analysis of the $P K D 1$ proximal promoter revealed a putative early growth response 1 (EGR1) binding site, which was confirmed by chromatin immunoprecipitation (ChIP) and quantitative PCR. Further analysis of publicly available ChIP-sequencing data revealed that this putative binding site overlapped with a conserved EGR1 binding peak present in 5 other cell lines. Quantitative real-time PCR showed that EGR1 suppressed PKD1, while Angll significantly up-regulated PKD1, an effect counteracted by pioglitazone. Conversely, in EGR1 short hairpin RNA lentivirally
\end{abstract}

transduced THP-1 cells, reduced EGR1 led to a significant upregulation of $P K D 1$, especially after treatment with pioglitazone. In vivo, deficiency of Egr1 in the haematopoietic compartment of mice completely abolished the incidence of $\mathrm{CaCl}_{2}$-induced aneurysm formation. @ 2015 S. Karger AG, Basel

\section{Introduction}

Abdominal aortic aneurysms (AAAs) affect the male more than the female population, and although mortality rates due to rupture have improved in the UK and the US, globally they remain a significant cause of death [1]. The precise aetiology of AAA is unknown; the disease is characterised by extensive vascular inflammation, smooth muscle cell apoptosis, oxidative stress and extracellular matrix remodelling, which all result in a dilatation of the aortic vessel [2]. Vascular inflammation is a complex and multistep process involving local activation of circulating monocytes and their infiltration into the vessel wall, where they differentiate to macrophages and secrete chemotactic factors and macrophage-activating cytokines that subsequently up-regulate matrix metalloproteinases (MMPs) and vascular adhesion molecules [3-5]. The chronic nature of this process requires further recruitment of monocytes into the vessel wall, promoting further degradation of the extracellular matrix by MMPs, driving progressive dilatation.

\section{KARGER 125}

C 2015 S. Karger AG, Base

$1018-1172 / 15 / 0522-0081 \$ 39.50 / 0$ 
Surgery is currently the only available treatment for AAA patients, although it is not always applicable or risk free. An effective drug therapy is greatly needed. We and others have shown that treatment with the peroxisome proliferator-activated receptor $\gamma$ agonists rosiglitazone and pioglitazone was able to regress aneurysm formation in the angiotensin II (AngII)-induced mouse model of AAA $[6,7]$. Pioglitazone was also shown to reduce macrophage infiltration in the vascular wall, an early feature of the AngII-induced aneurysms [6-9]. An effective nonsurgical therapy that could inhibit this process may have a significant impact on AAA growth and stabilisation.

Since a recent meta-analysis of randomised controlled trials using thiazolidinediones resulted in their effective withdrawal, due to increased cardiovascular risk [10], we used pioglitazone to identify new drug targets for the therapeutic treatment of aneurysm. We performed expression profiling of phorbol ester-differentiated THP-1 monocytic leukaemia cells that were treated with AngII or with AngII and pioglitazone. The polycystic kidney disease 1 (PKD1) gene was among the genes that were significantly up-regulated genomewide after multiple testing in our expression profile. Mutations in the $P K D 1$ gene are the main cause of autosomal dominant polycystic kidney disease (ADPKD), which is also linked to an increased incidence of aneurysms in human and mouse models [11-14]. Exploring its regulation further, we found that it is an early growth response 1 (EGR1) target gene, with EGR1 being a functional suppressor of PKD1. EGR1 is immediately up-regulated during monocytic differentiation and recognises $\mathrm{G} / \mathrm{C}$-rich reach sequences in promoters of active genes [15]. Additionally, we show that AngII up-regulates $P K D 1$ expression, that pioglitazone can ameliorate this effect and that removal of Egrl from the haematopoietic compartment of mice abolishes aneurysm formation in a $\mathrm{CaCl}_{2}$-induced AAA experimental murine model.

\section{Materials and Methods}

\section{Cells and Treatment}

THP-1 cells (ATCC, LGC standards, Teddington, UK) were maintained in RPMI 1640 (Sigma-Aldrich, Dorset, UK) supplemented with $10 \%(\mathrm{v} / \mathrm{v})$ foetal bovine serum (Gibco, Life Technologies, Paisley, UK) and $1 \%(\mathrm{v} / \mathrm{v})$ penicillin/streptomycin (SigmaAldrich, Dorset, $\mathrm{UK}$ ) at $37^{\circ} \mathrm{C}$ in a $5 \% \mathrm{CO}_{2}$-humidified incubator. Suspension THP-1 cultures were passaged at a ratio of $1 / 10$ twice weekly. For experiments, the THP-1 cells were counted, seeded at $1 \times 10^{6} \mathrm{cells} / \mathrm{ml}$ and differentiated to macrophages in the presence of $100 \mathrm{nM}$ phorbol 12-myristate 13-acetate (PMA; Sigma-Aldrich) for $4 \mathrm{~h}$. After this 4-hour period, when the cells became adherent to the plate, PMA was washed off, and the monolayer was placed in growth medium (GM) alone (control cells), in GM with AngII (1 $\mathrm{mg} / \mathrm{ml}$; Bachem, Bubendorf, Switzerland) and in GM with AngII $(1 \mathrm{mg} / \mathrm{ml})$ and pioglitazone $(1 \mu \mathrm{M}$; Sigma-Aldrich). Thereafter, the monolayers were harvested at 12,24 and $48 \mathrm{~h}$.

\section{Microarray}

The THP-1 cells were differentiated to macrophages by addition of PMA (100 nM) for $4 \mathrm{~h}$. The control THP-1 cells remained in the PMA, and the treatment cells were supplemented with AngII (1 mg/ $\mathrm{ml})$ or AngII $(1 \mathrm{mg} / \mathrm{ml})$ and pioglitazone $(1 \mu \mathrm{M})$ for another $24 \mathrm{~h}$. At this time point, all cells, the PMA controls and the treated macrophages, were harvested for total RNA isolation with TRIzol ${ }^{\mathrm{TM}}$ (Life Technologies, Paisley, UK) according to the manufacturer's instructions. Biotinylated, high-quality amplified antisense RNA was prepared using the Illumina ${ }^{\circledR}$ TotalPrep RNA amplification kit (Life Technologies, Paisley, UK) following the manufacturer's recommendations. The antisense RNA samples were hybridised to HumanHT-12 expression arrays (Illumina, Saffron Walden, UK). Subsequent staining of the hybridised arrays was performed with streptavidin-Cy3 (GE Healthcare, Life Sciences, Little Chalfont, UK) according to the manufacturer's recommendation (Whole Genome Gene Expression Direct Hybridisation assay; Illumina), and the arrays were scanned with an Illumina BeadStation GX500 (Illumina). The samples consisted of 3 experimental repeats for the following treatments: PMA only THP-1 cells, PMA and AngII-treated cells, and PMA and AngII/pioglitazone-treated cells. The data obtained from the chip readouts were adjusted using quantile normalisation and were analysed with the GeneSpring GX software. Statistically significant (i.e. $\mathrm{p}<0.05)$ differentially expressed genes were identified using one-way analysis of variance (ANOVA) with the Tukey HSD post hoc test and multiple testing with the Benjamini-Hochberg for false discovery rate correction. Raw array data are accessible through the ArrayExpress database (EMBL-EBI; E-MTAB-2320).

\section{Chromatin Immunoprecipitation with Quantitative PCR}

Chromatin immunoprecipitation (ChIP) was performed as previously described [16]. Briefly, $8 \times 10^{7}-1 \times 10^{8}$ treated THP-1 cells were cross-linked with a solution of $1 \%(\mathrm{v} / \mathrm{v})$ formaldehyde for 10-20 min at room temperature. The cell pellets were then washed and resuspended in a series of lysis buffers. Sonication at maximum output power $\left(26 \mathrm{~W} / \mathrm{cm}^{2}\right)$ at $4{ }^{\circ} \mathrm{C}$, for a total processing time of $7 \mathrm{~min}(14 \times 30 \mathrm{~s}$ sonication sessions with 1 min rest intervals in between), was applied to obtain DNA fragments of $0.2-$ $1.0 \mathrm{~kb}$. From the resulting sonicated whole cell extract, a portion (1/38.5) was kept as input, and the rest was incubated overnight with Dynal Protein G magnetic beads (Life Technologies) coated either with $10 \mu \mathrm{g}$ of EGR1 antibody (44D5; Cell Signaling Technology, New England Biolabs, Hitchin, UK) or normal rabbit IgG control antibody (2,729; Cell Signaling Technology, New England Biolabs). The beads were washed 6 times with RIPA buffer (Cell Signalling Technology, Danvers, USA) and 1 final time with TE/50 mM $\mathrm{NaCl}$. Bound antibody-chromatin complexes were eluted at $65^{\circ} \mathrm{C}$ with brief vortexing every $10 \mathrm{~min}$ for $1 \mathrm{~h}$. The input chromatin and antibody-chromatin complexes were further incubated at $65^{\circ} \mathrm{C}$ for $6 \mathrm{~h}$ for the reversal of the cross-links. The sonicated input DNA and the immunoselected DNA were then treated with RNase A and proteinase $\mathrm{K}$ and extracted with phenol:chloroform:isoamyl alcohol (24:24:1; Sigma-Aldrich). Purified DNA was then blunted with T4 DNA polymerase (Promega, Chilworth, UK), ligated to linker sequences and subjected to 2 sequential protocols of linker-medi- 
ated PCR (online suppl. table 1; for all online suppl. material, see www.karger.com/doi/10.1159/000430986). EGR1 occupancy at the PKD1 promoter was analysed ChIP and quantitative PCR (ChIP-qPCR) using SYBR green chemistry (Bio-Rad, Hemel Hempstead, UK) and a CXF384 Touch cycler (Bio-Rad; primers and conditions are listed in online suppl. table 2). Serial dilutions of input DNA were used for the generation of a standard curve. The ChIP DNA was loaded at 1/10 of the highest input concentration, and the cycle threshold values after subtraction of the signals for the control IgG antibody were used to calculate the percentage of recovery relative to the input DNA.

\section{EGR1 Binding Site Analysis}

The sequence of the promoter $( \pm 1,500-2,000 \mathrm{~kb}$ from the transcriptional start site) of the human polycystin-1 gene, PKD1 (ENSG00000008710), was accessed through the Ensembl human genome browser (www.ensembl.org/Homo_sapiens/Info/Index). The sequence was copied to the online tool for the search of putative transcription factor binding sites, TFSEARCH (www.cbrc.jp/research/ $\mathrm{db} /$ TFSEARCH.html), and the executed search returned, among other transcription-factor putative binding sites, an EGR1 binding site with a score of $94.5 \%$ within the proximal promoter of PKD1 (-322 bp from the transcription start site (TSS)). Primer sets were designed around this locus in order to determine EGR1 binding to this region. A primer set binding to a nearby locus was also designed to account for a locus-specific negative control (online suppl. fig. 1, table 2).

\section{Publicly Available EGR1 ChIP-Sequencing Data}

Duplicate sets of publicly available EGR1 ChIP-sequencing (ChIP-seq) data from 6 cell lines (Eccl endometrial epithelial cells, Gm12878 EBV-transformed B lymphocytes, H1 human embryonic stem cells, Hct116 colorectal carcinoma, K562 erythrocytes and Mcf7 mammary epithelial cells) were downloaded from the NCBI GEO depository [17] (http://www.ncbi.nlm.nih.gov/ geo/query/acc.cgi?acc = GSE32465; as part of the ENCODE (ENCyclopedia Of DNA Elements) project). The GEO samples IDs and method of access and analyses of the EGR1 peaks are included in the online supplementary information, section 1.2.

RNA Extraction, cDNA and Quantitative Real-Time PCR

For cDNA synthesis, the RNA was isolated from the THP-1 cells treated as above, using the RNeasy Mini Kit (Qiagen, Manchester, UK) with on-column DNase I digestion according to the manufacturer's instructions. Then, up to $3 \mu \mathrm{g}$ of input RNA were randomly primed and reverse transcribed to cDNA using the GoScript Reverse Transcription System (Promega) following the manufacturer's recommendations. The selection of the most stably expressed reference genes for the THP-1 cells under the experimental conditions used for this work was done with quantitative real-time PCR (qRT-PCR) using the geNorm Reference Gene selection kit (Primerdesign, Rownhams, UK). The 12 genes included in the kit are shown in figure 2 of the online supplementary information. The results were analysed with the $\mathrm{qBase}^{+}$software (Biogazelle, Zwinjnaarde, Belgium). The mitochondrial succinate dehydrogenase (SDHA) and phospholipase A2 (YWHAZ) genes were found to be the most stably expressed reference genes in the THP-1 cells (online suppl. fig. 2 and table 3 ), and therefore, they were used for the normalisation of the gene expression. The EGR1 and PKD1 expressions were validated by probe-based $\mathrm{qRT}$-PCR prepared in duplicates from 3 independent experimental repeats. In each
qRT-PCR, $25 \mathrm{ng}$ (in $5 \mu \mathrm{l}$ ) of each cDNA sample were amplified using $1 \mu \mathrm{l}$ of each double-dye-based probe, $10 \mu \mathrm{l}$ of GoTaq Probe qPCR Master mix (Promega) and $5 \mu$ l of nuclease-free water under universal thermal cycling conditions (online suppl. table 3 ) in a CFX384 Touch cycler (Bio-Rad). The gene expression data were normalised against the average expression of the above reference genes and analysed using the comparative cycle threshold method.

\section{Immunofluorescence}

The THP-1 cells were grown in chamber slides (Nunc Lab-Tek Permanox, Fisher Scientific, Loughborough, UK) and treated as described above. After 24 or $48 \mathrm{~h}$ of treatment, the cells were washed twice with phosphate-buffered saline (PBS; Sigma-Aldrich), fixed with $4 \%(\mathrm{v} / \mathrm{v})$ paraformaldehyde (Polysciences Inc., Park Scientific Ltd., Moulton Park, UK)/PBS at room temperature for $10 \mathrm{~min}$ and washed again twice with PBS. The cells were permeabilised with $0.1 \%$ Triton X100 (Sigma-Aldrich) in PBS for $5 \mathrm{~min}$ and washed twice with PBS. Blocking was performed in $20 \%$ foetal calf serum/PBS for $30 \mathrm{~min}$ at room temperature. The cells were incubated with rabbit polyclonal antibody to polycystin-1 (H-260; Santa Cruz sc-25570, Insight Biotechnology, Wembley, UK) followed by swine anti-rabbit FITC IgG (F0205; Dako, Cambridge, UK). The slides were counterstained with DAPI, mounted with aqueous mounting medium (ab128982; Abcam, Cambridge, UK) and visualised with a Zeiss Axiovert inverted fluorescence microscope (Zeiss, Jena, Germany). The images were acquired with a Leica TCS camera (Leica, Solms, Germany) using Zeiss AxioVision software (Zeiss).

\section{RNA Interference}

The freshly grown THP- 1 cells were counted, and $5 \times 10^{4}$ cells were seeded in each well of a 24 -well plate in $500 \mu$ of GM with the transduction adjuvant polybrene $(8 \mu \mathrm{g} / \mathrm{ml}$; Santa Cruz, Insight Biotechnology). EGR1 short hairpin RNA (shRNA) (sc-29303-V; Santa Cruz, Insight Biotech) lentiviral particles $(5,000 \mathrm{IFU} / \mu \mathrm{l})$ that carry constructs encoding 3-5 target-specific shRNAs and a puromycin antibiotic resistance cassette were used at a multiplicity of infection of 5:1. After $24 \mathrm{~h}$, the polybrene was removed, and the cells were placed in GM and allowed to grow overnight. Next, the cells were split at a 1/5 ratio and allowed to grow further for $48 \mathrm{~h}$. The selection of transduced clones was done with $2 \mu \mathrm{g} / \mathrm{ml}$ puromycin dihydrochloride (Santa Cruz, Insight Biotechnology), as determined by a puromycin killing curve. Thereafter, the medium was replaced every 3-4 days until surviving, growing, stably transduced THP-1 clones were selected.

\section{Bone Marrow Chimeric Mice Generation}

Male donor (8-week-old) homozygous deficient mice (B6N;129Egr $1^{\text {tm1Jmi/J }}$ ) (Jackson Laboratory, Maine, USA) were killed, and their femurs and tibias were removed aseptically. The marrow cavities were flushed out with $\mathrm{Ca}^{2+} / \mathrm{Mg}^{2+}$-free Hank's basal salt solution (Gibco), using a 25-gauge needle attached to a 1-ml syringe. Singlecell suspensions were prepared by repeated pipetting, and the resulting cell preparation was passed through a nylon mesh to remove particulate matter. The cells were washed twice in Hank's balanced salt solution, counted using a haemocytometer and resuspended at $3 \times 10^{7}$ cells $/ \mathrm{ml}$ in RPMI 1640 before transplantation. Eight-weekold female wild-type litter mate recipient mice received a lethal dose of whole-body X-irradiation (950 rads). The irradiated recipients received $1 \times 10^{7}$ cells in $0.3 \mathrm{ml}$ RPMI 1640 (Gibco) within $6 \mathrm{~h}$ of the 
irradiation via a tail vein injection. The repopulation was confirmed using fluorescence in situ hybridisation (FISH) for the Y chromosome (see below). $\mathrm{CaCl}_{2}$-induced aneurysms were generated in these chimeric mice 16 weeks after the bone marrow transfer.

\section{Fluorescence in situ Hybridisation}

Blood smears from the chimeric mice were collected at sacrifice, and the $\mathrm{Y}$ chromosome of the donor marrows was detected using a Y chromosome painting kit (Cytocell, Cambridge, UK) according to the manufacturer's recommendations. The resulting staining was visualised using a Zeiss Axiovert inverted fluorescence microscope, and the images were acquired with a Leica TCS camera using the Zeiss AxioVision software.

\section{$\mathrm{CaCl}_{2}$-Induced Aneurysm Formation}

All animal experiments were conducted with local ethical approval and reported according to the ARRIVE guidelines (https:// www.nc3rs.org.uk/arrive-guidelines). The $\mathrm{CaCl}_{2}$-induced model experiments were conducted using a number of minor modifications tothose previouslyreported [18]. Mice(B6N;129-Egr $\left.1^{\mathrm{tm} 1 \mathrm{Jmi} / \mathrm{J}}\right)$, aged 24 weeks, were anaesthetised to the surgical plane as described in the ARRIVE guidelines, then prepared for sterile surgical procedure and laparotomised $2-5 \mathrm{~mm}$ to the left of a central line. Abdominal aneurysmal dilation was induced ( $n=10$ /group) between the renal arteries and iliac bifurcation following the measurement of the aortic diameter $(\mu \mathrm{m})$ using computer-assisted micrometry ( $n=5 /$ animal) by an operator independent of the treatment. Dilation was induced by the application of 2 consecutive Whatmann ${ }^{\mathrm{TM}} 3 \mathrm{MM}$ (GE Healthcare) rectangles (cut to the size of the artery), saturated with sterile $0.25 \mathrm{M} \mathrm{CaCl}_{2}$ (Sigma-Aldrich) for $7 \mathrm{~min}$ (a total application time of $14 \mathrm{~min}$ ). Excess $\mathrm{CaCl}_{2}$ was then removed by swabbing the area with cotton buds soaked in $0.9 \%$ sterile saline prior to the closure of the wound. The mice were given an anaesthetic reversal agent (as described, ARRIVE report, suppl. file), and fully recovered before returning to the maintenance cages for 6 weeks. The animals were observed twice a day for signs of condition and weighed on alternate days to monitor their food intake. At 6 weeks after the induction, the animals were sacrificed by a lethal overdose of anaesthesia and cardiac puncture.

\section{Statistical Analysis}

The results are expressed as means \pm standard error of the mean or as means \pm standard deviation, as indicated. One-way ANOVA was performed using the GraphPad Prism 5 software with statisti$\mathrm{cal}$ significance assessed at $\mathrm{p}<0.05$. Aneurysm incidence was compared using Student's t test.

Fig. 1. Microarray-based expression profiling of PMA-differentiated THP-1 cells after AngII, AngII/pioglitazone or no treatment control. a Overview of the gene expression changes organised by hierarchical clustering. The genes are sorted by functional relevance; change in expression is shown by colour (yellow, unchanged; blue, down-regulated; red, up-regulated expression), and the level of change is represented by the colour intensity. The experimental triplicates for each treatment are shown with AngIItreated THP-1 cells on the left (AngII), AngII/pioglitazone in the middle $(\mathrm{A}+\mathrm{P})$ and no treatment controls (reference condition) on the right (Nil). b Focus on a gene cluster where the expression

\section{Results}

\section{Microarray Profiling of PMA-Differentiated THP-1}

Cells

The global gene expression changes in the macrophages were identified using a microarray-based expression profiling. The THP1 cells were differentiated with PMA for $4 \mathrm{~h}$ followed by AngII, AngII/pioglitazone or no treatment control for a further $24 \mathrm{~h}$. Expression profiling confirmed changes after this period, also reported elsewhere, that were relevant to the treatments applied, e.g. AngII treatment significantly up-regulated the expression of genes, such as MMP1 [19] and MMP12 [4]. Pioglitazone modulated the expression of genes that are known targets of peroxisome proliferator-activated receptor $\gamma$, such as FABP4 [20] along with others (fig. 1 and online suppl. table 4). This initial analysis indicated that cultured PMA-differentiated THP-1s, followed by treatment with AngII or AngII and pioglitazone can recapitulate changes previously described for inflammatory cells in an aneurysmal setting (see gene ontological and pathway analyses, online suppl. fig. 3 and table 5). PKD1 was found to be among the genes significantly modulated in a genomewide analysis (using a corrected $\mathrm{p}$ value cut-off of $\mathrm{p}<0.05$ after applying a Benjamini-Hochberg multiple testing correction) after treatment with AngII or AngII and pioglitazone. The involvement of PKD1 in the increased incidence of aneurysms led to the following investigations.

\section{A Putative EGR1 Binding Site at the Promoter of PKD1 Is Occupied by EGR1 after PMA-Induced Macrophage Differentiation and during Treatment with AngII or AngII and Pioglitazone}

The differentiation of THP-1 cells to macrophages is concomitant with an abrupt and immediate up-regulation of the EGR1 protein, which returns to just detectable levels $48 \mathrm{~h}$ following the differentiation (online suppl.

changed due to treatment with AngII, but the presence of pioglitazone abolished these effects (gene expression is comparable to the Nil reference condition). c Focus on a gene cluster where the expression changed due to treatment with pioglitazone, while AngII had no effect, with gene expression being comparable to the Nil reference condition. Arrows point at MMP1, MMP12 and $F A B P 4$. The number of up-regulated/down-regulated genes and the overview ontology output (WEGO) and pathway analyses (Panther) are shown in the online supplementary tables 4 and 5, and in online figure 3 . The raw array data are accessible through ArrayExpress (EMBL-EBI; E-MTAB-2320).

(For figure see next page.)
Charolidi/Pirianov/Torsney/Pearce/ Laing/Nohturfft/Cockerill 


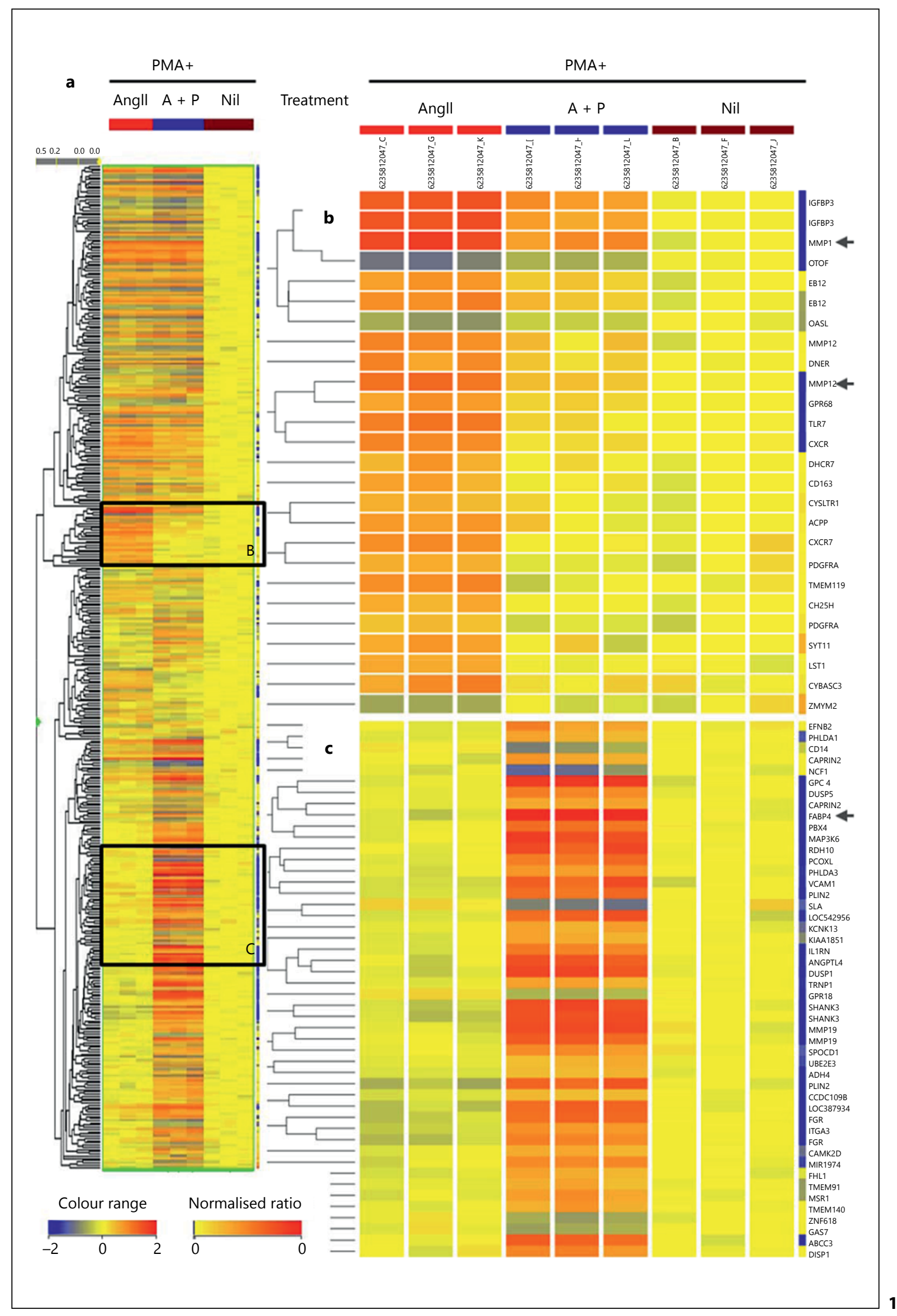



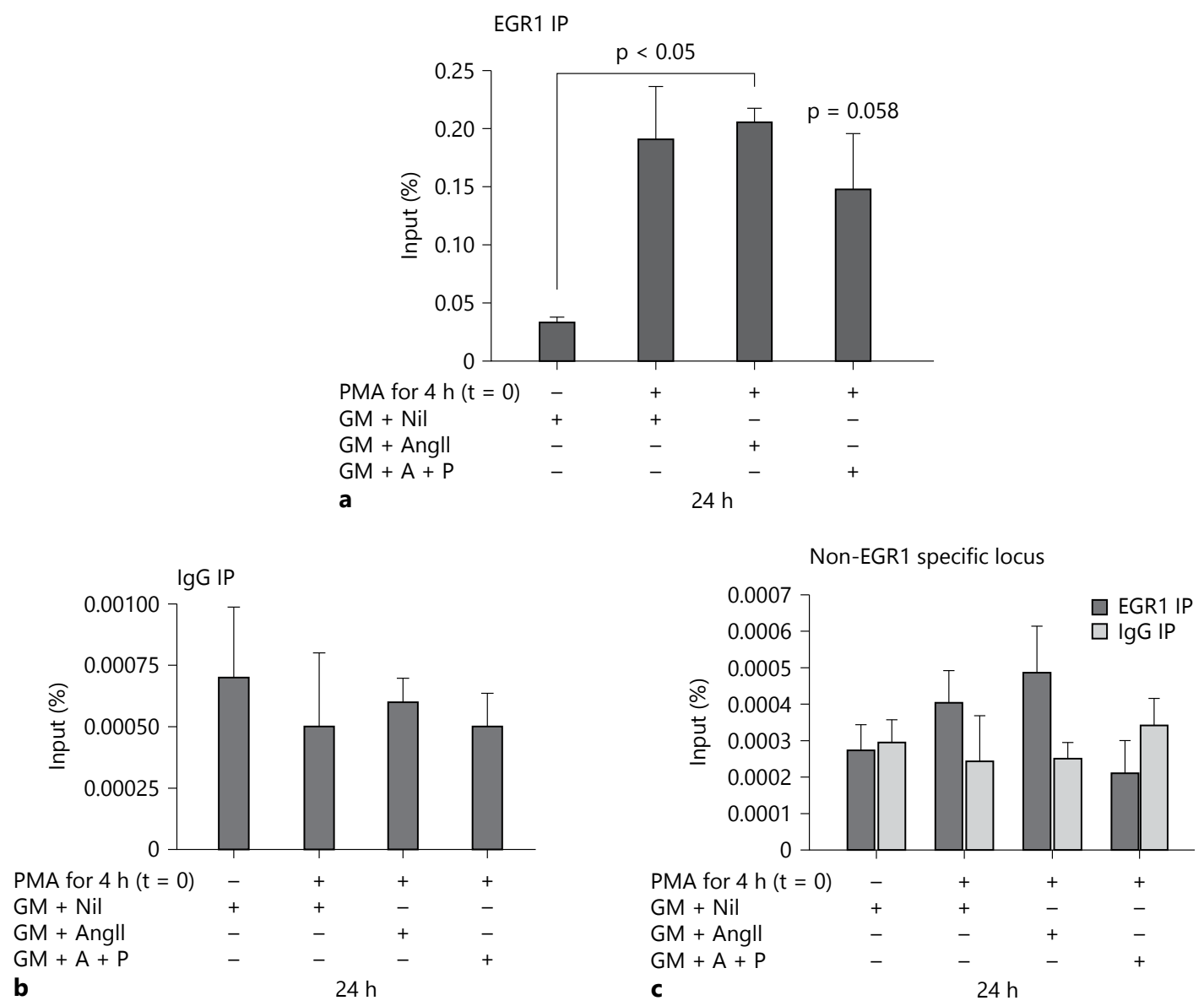

Fig. 2. EGR1 binds at the $P K D 1$ proximal promoter. a The percentage of recovery relative to input chromatin was measured using qPCR with primers flanking the putative EGR1 binding site. The binding of EGR1 at the EGR1 consensus of the PKD1 promoter was confirmed by the recovery of immunoprecipitated fragments for all 3 conditions tested after PMA-induced differentiation, no treatment $(\mathrm{GM}+\mathrm{Nil})$, AngII $(\mathrm{GM}+$ AngII $)$ and AngII/pioglitazone $(\mathrm{GM}+\mathrm{A}+\mathrm{P})$. Undifferentiated THP-1 cells (GM only) express just detectable levels of EGR1, and therefore, this condition

fig. 4). The PKD1 promoter harbours a putative EGR1 binding site in its proximal promoter, 322 bp upstream of the TSS (online suppl. fig. 1). To determine whether EGR1 occupies this promoter region, we performed ChIP-qPCR. Immunoprecipitation of the cross-linked cells after PMA-induced differentiation and AngII, An$\mathrm{gII} /$ pioglitazone or no treatment control was performed with an EGR1 antibody, and the analysis of the immunoprecipitated fragments was achieved with primers flanking the putative EGR1 binding site (online suppl. fig. 1). The percent recovery of the immunoprecipitates rele- was used as a negative control of EGR1 recovery from the EGR1 binding locus and for comparison with the other conditions. b Non-specific background recovery was very low, as measured by ChIP using an IgG antibody. c Primers designed around a nonEGR1-specific locus within the proximal promoter of PKD1 also showed very low recovery, confirming the specificity of the EGR1 recovery. The data represent the means \pm standard deviation from 3 independent experiments with $p$ values as indicated.

vant to input (non-immunoprecipitated chromatin) is shown in figure 2a. Undifferentiated THP-1 cells express marginally detectable levels of EGR1, and therefore, they were used as a negative control for EGR1 recovery from the $P K D 1$ promoter (i.e. $0.033 \pm 0.005 \%$ of input). The percent recovery after $24 \mathrm{~h}$, for all 3 conditions after PMA differentiation, AngII, AngII/pioglitazone and no treatment, ranged from $0.148 \pm 0.064$ (AngII/pioglitazone) to $0.205 \pm 0.012 \%$ of input (AngII), confirming that EGR1 occupies its consensus site at the PKD1 promoter. The comparison of the levels of recovery from 
Fig. 3. Analyses of available ChIP-seq data reveal a conserved EGR1 binding peak within the PKD1 proximal promoter of multiple cell lines. a Duplicate EGR1 binding peak coordinates for PKD1 were extracted from NCBI GEO (accession number GSE32465; part of the ENCODE project). Overlapping peaks were merged and graphically aligned to genomic loci (hg19) using $\mathrm{R}$ code based on Gviz and ggplot2 packages. A common conserved EGR1 binding peak within the PKD1 proximal promoter was identified in the above cell lines. The widths in the bar graphs indicate the position and heights proportional to the average 'score' values provided by ENCODE data. b Sequence of the conserved EGR1 binding peak within the $P K D 1$ proximal promoter. $\mathrm{c}$ Four out of the above 5 cell lines also harbour EGR1 binding peaks within the $P K D 2$ promoter.

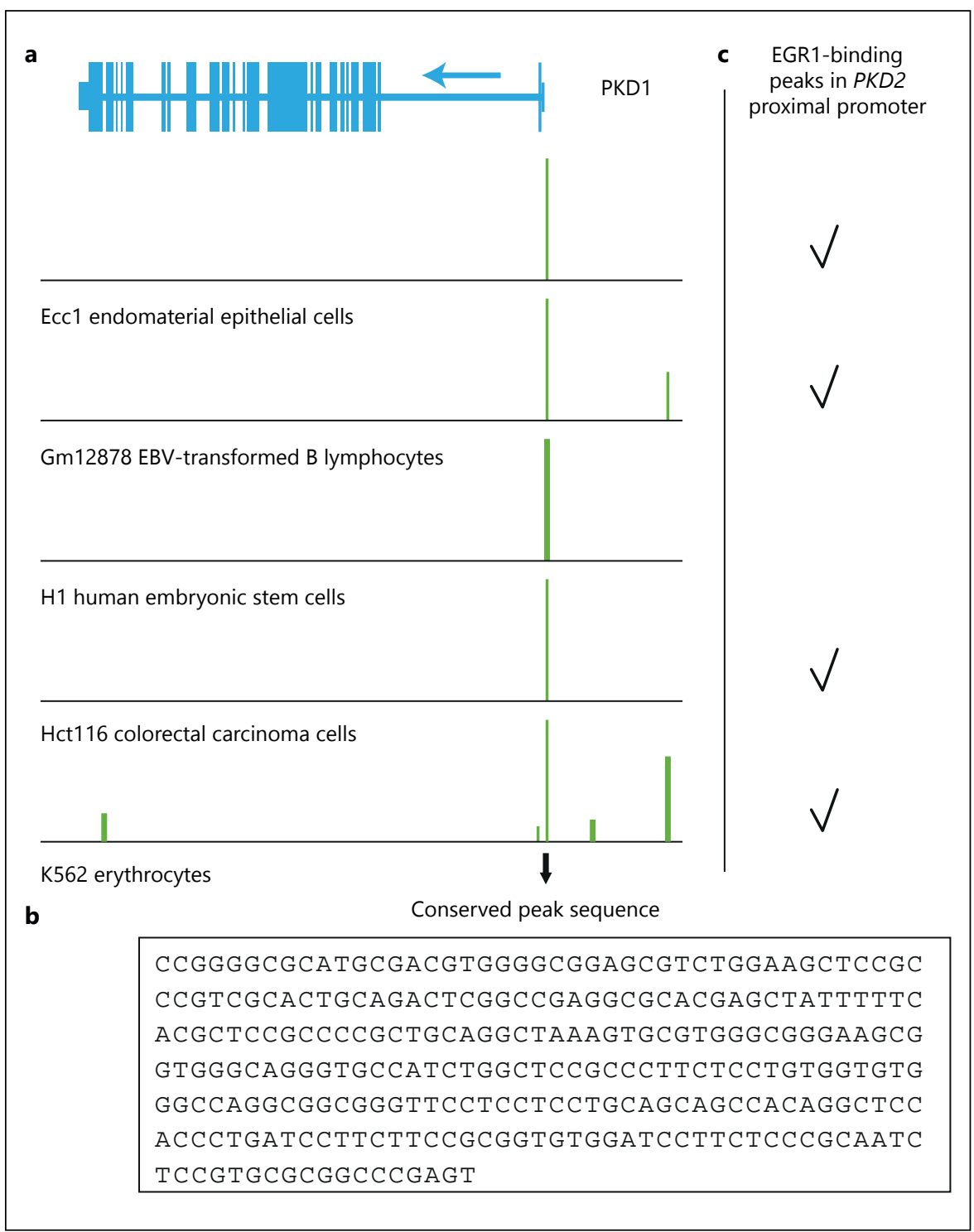

AngII, AngII/pioglitazone or no treatment with the recovery from undifferentiated THP-1 cells showed that recoveries from AngII and no treatment conditions were significant $(\mathrm{p}<0.05)$, but not from AngII/pioglitazone $(\mathrm{p}=0.058)$. A low background ChIP noise was confirmed with IgG immunoprecipitation under the same conditions (fig. 2b). In addition, in order to determine whether EGR1 localisation was specific and not an artefact of the ChIP-qPCR procedure, EGR1 recovery was assessed with primers flanking a nearby locus of the PKD1 promoter (1,074 bp downstream from TSS, online suppl. fig. 1). The very low levels of recovery (fig. 2c) from both EGR1 and IgG immunoprecipitants confirmed the specificity of the ChIP-qPCR technique. In PMA-differenti- ated THP-1 cells, after AngII or no treatment, EGR1 occupancy at the promoter of PKD1 was higher, but lower after AngII/pioglitazone treatment.

\section{EGR1 Plays a Role in PKD1 Expression}

In order to explore whether EGR1 binds at the PKD1 promoter in other cell types, we interrogated publicly available EGR1 ChIP-seq data from the ENCODE project (NCBI GEO, GSE32465). One common EGR1 peak upstream of $P K D 1$ was identified in 5 out of 6 lines for which data were available, including Eccl endometrial epithelial cells, Gm12878 EBV-transformed B lymphocytes, H1 human embryonic stem cells, Hct 116 colorectal carcinoma and K562 erythrocytes (fig. 3a). Common to the 5 
peaks was a segment of $276 \mathrm{bp}$ (fig. 3b) that is located 203 bp upstream of the PKD1 TSS and encompasses the EGR1 consensus element discussed earlier (online suppl. fig. 1). No EGR1 peak was identified in Mcf7 mammary epithelial cells. In addition, 4 out of these 6 cell lines (except the Mcf7 mammary epithelial and the H1 human embryonic stem cells) harbour EGR1 binding peaks within their PKD2 gene promoter (fig. 3c). Taken together, EGR1 binds at the PKD1 proximal promoter of multiple cell types, supporting a regulatory role for EGR1 in PKD1 expression.

\section{EGR1 Suppresses PKD1 Expression during}

Differentiation, AngII Up-Regulates the Expression of

PKD1 and Pioglitazone Ameliorates This Effect

In order to determine the effect of EGR 1 binding at the $P K D 1$ promoter, we checked the expression of $P K D 1$ in PMA-differentiated THP-1 cells after AngII, AngII/pioglitazone or no treatment control for 12, 24 and $48 \mathrm{~h}$ by qRT-PCR. Increased expression of EGR 1 coincided with lower expression of $P K D 1$, and reduced expression of EGR1 with increased expression of $P K D 1$ over time, suggesting that EGR1 may suppress $P K D 1$ expression in differentiating THP-1 cells (fig. 4a, b). Treatment with AngII significantly up-regulated the expression of $P K D 1$ by $24 \mathrm{~h}$ $(\mathrm{p}<0.0001)$ and $48 \mathrm{~h}(\mathrm{p}<0.01)$. Parallel treatment with pioglitazone ameliorated the effect of AngII, and the expression levels of $P K D 1$ were comparable to those in the no-treatment differentiated THP-1 cells for both 24 and $48 \mathrm{~h}$. At the protein level, the expression of the PKD1 product, polycystin-1, was assessed by immunocytochemistry under the same conditions. Polycystin-1 was present in all 3 conditions for both time points. A representative staining after $48 \mathrm{~h}$ of treatment is shown in figure 4c. Polycystin-1 staining was highly comparable between the no-treatment controls and the AngII/pioglitazone-treated cells and less prominent than in AngII-treated cells. These data in combination with the lower EGR1 occupancy at the $P K D 1$ promoter after pioglitazone treatment (fig. 2a) strongly suggest that regulation of $P K D 1$ expression under these conditions may involve other factor(s) and/or binding element(s) present in the $P K D 1$ promoter.

\section{EGR1 shRNA Lentivirally Transduced THP-1 Cells}

Express Higher Levels of PKD1

In order to verify the function of EGR1 binding at the PKD1 promoter, the THP-1 cells were stably transduced with lentiviral EGR1 shRNA for silencing the EGR1 expression. The undifferentiated EGR1 shRNA lentivirally transduced THP-1 cells showed no obvious phenotypic change from the non-virally transduced THP-1 cells. However, the PMA-induced differentiation of virally transduced THP-1 cells required a PMA-incubation 3 times longer for the cells to become adherent (12 instead of $4 \mathrm{~h}$ ). PKD1 expression in the EGR1 shRNA lentivirally transduced cells was significantly higher $(\mathrm{p}<0.0001)$ than that found in control non-virally transduced cells (fig. 5a), as shown by qRT-PCR. The EGR1 expression was significantly depleted in the EGR1 shRNA virally transduced THP- 1 cells ( $\mathrm{p}<0.0001$; fig. 5b). Elevated levels of EGR1 seem to be required early on (initial time points), as low levels of $E G R 1$ by $48 \mathrm{~h}$ did not affect $P K D 1$ expression (no treatment controls, $48 \mathrm{~h}$; fig. 5a). However, a lack of $E G R 1$ at the initial time points reversed the effects of AngII and AngII/pioglitazone treatments in the EGR1 shRNA lentivirally transduced THP-1 cells. In those THP-1 cells where EGR1 had been silenced, AngII failed to induce an up-regulated $P K D 1$ expression as it did when EGR1 was present, and AngII/pioglitazone treatment induced a significantly higher PKD1 expression. These data further suggest that EGR1 may co-operate with additional factors for the fine-tuning of $P K D 1$ expression.

Deficiency of Egr1 in the Haematopoietic

Compartment Inhibits Aneurysm Formation in a

$\mathrm{CaCl}_{2}$-Induced Murine Model

We used an AngII-independent model to explore the role of Egr1 in aneurysm aetiology. We constructed bone marrow chimeric mice in which the haematopoietic compartment was reconstituted using marrow from homozygous deficient Egr 1 mice in wild-type litter mates following bone marrow ablation. Control chimeric mice, reconstituted with wild-type bone marrow, produced $50 \%$ aneurysms following the aortic application of $0.25 \mathrm{M}$ $\mathrm{CaCl}_{2}$, whereas in mice with Egr1-deficient bone marrow no aneurysms were observed $(\mathrm{n}=10, \mathrm{p}=0.048$; table 1$)$.

\section{Discussion}

Our findings support the role of macrophages in aneurysm development and define EGR1 as a key gene in this process. In the present study, we are showing that (1) EGR1 localises at the PKD1 proximal promoter; (2) AngII induces expression of $P K D 1$ in differentiating macrophages, while pioglitazone ameliorates this effect; (3) EGR1 is a functional suppressor of $P K D 1$ expression, and (4) Egrl is necessary for $\mathrm{CaCl}_{2}$-induced aneurysm formation.
88

J Vasc Res 2015;52:81-93 DOI: $10.1159 / 000430986$
Charolidi/Pirianov/Torsney/Pearce/ Laing/Nohturfft/Cockerill 
Fig. 4. Expression of $P K D 1$ and $E G R 1$ in THP-1 cells. Relative RNA expression of PKD1 (a) and EGR1 (b). a, b The THP-1 cells were induced for 4 hours with PMA $(t=0)$, then the PMA was washed out and AngII (GM + AngII), AngII/pioglitazone $(\mathrm{GM}+\mathrm{A}+\mathrm{P})$ or no treatment control $(\mathrm{GM}+\mathrm{Nil})$ were applied for the time points indicated on the $\mathrm{x}$-axis. No treatment control THP-1 cells after 24 h were set as reference for comparison with other treatments/time points (relative expression $=1$ ). Data represent the means \pm standard error of the mean from 3 independent experiments with $\mathrm{p}$ values as indicated. Rel. = Relative; arb. = arbitrary. $\mathbf{c}$ Representative staining after $48 \mathrm{~h}$ of treatment. Polycystin-1 expression was assessed by immunocytochemistry under the same conditions. Polycystin-1 was localised at the cell periphery (arrowheads). The data are representative of 3 independent experiments, with i-iii representing $100 \%$ magnifications of the boxed areas in corresponding merged images; scale bars as indicated.

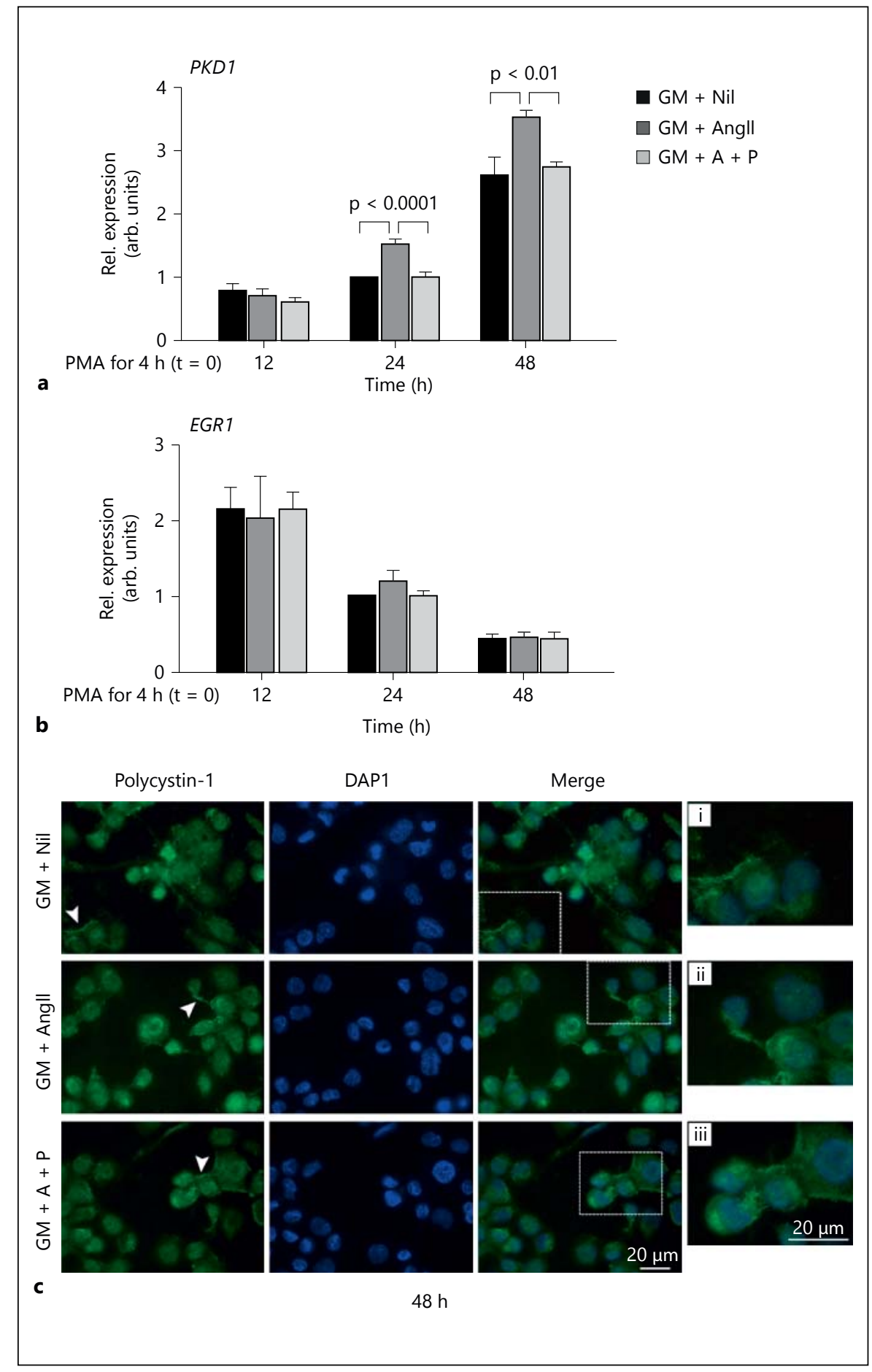

Monocyte differentiation is accompanied by changes in expression and requires the involvement of multiple transcription factors, e.g. EGR1 [15, 21], an immediate early induced factor. EGR1 binds to DNA G/C-rich sequences through 3 zinc-finger motifs in its carboxy ter- minal and modulates gene transcription through co-operation with other activating or repressing factors $[15,22$, 23]. Experimental evidence has linked EGR1 induction to chronic vascular inflammation, and it is highly expressed in the intraluminal thrombus of AAA patients [23-26]. 
Fig. 5. Expression of $P K D 1$ and EGR1 in EGR1 shRNA lentivirally transduced THP1 cells. Relative expression of PKD1 (a) and EGR1 (b). The THP-1 cells were induced for $12 \mathrm{~h}$ with PMA $(\mathrm{t}=0)$, then PMA was washed out and no treatment control (Nil), AngII (AngII) or AngII/pioglitazone (A + P) were applied for the time points indicated on the $\mathrm{x}$-axis. No treatment control THP- 1 cells after $24 \mathrm{~h}$ were set as reference for comparison with other treatments/time points (relative expression $=1$ ). Data represent the average \pm standard error of the mean from 3 independent experiments with $\mathrm{p}$ values as indicated. Rel. = Relative; arb. $=$ arbitrary.

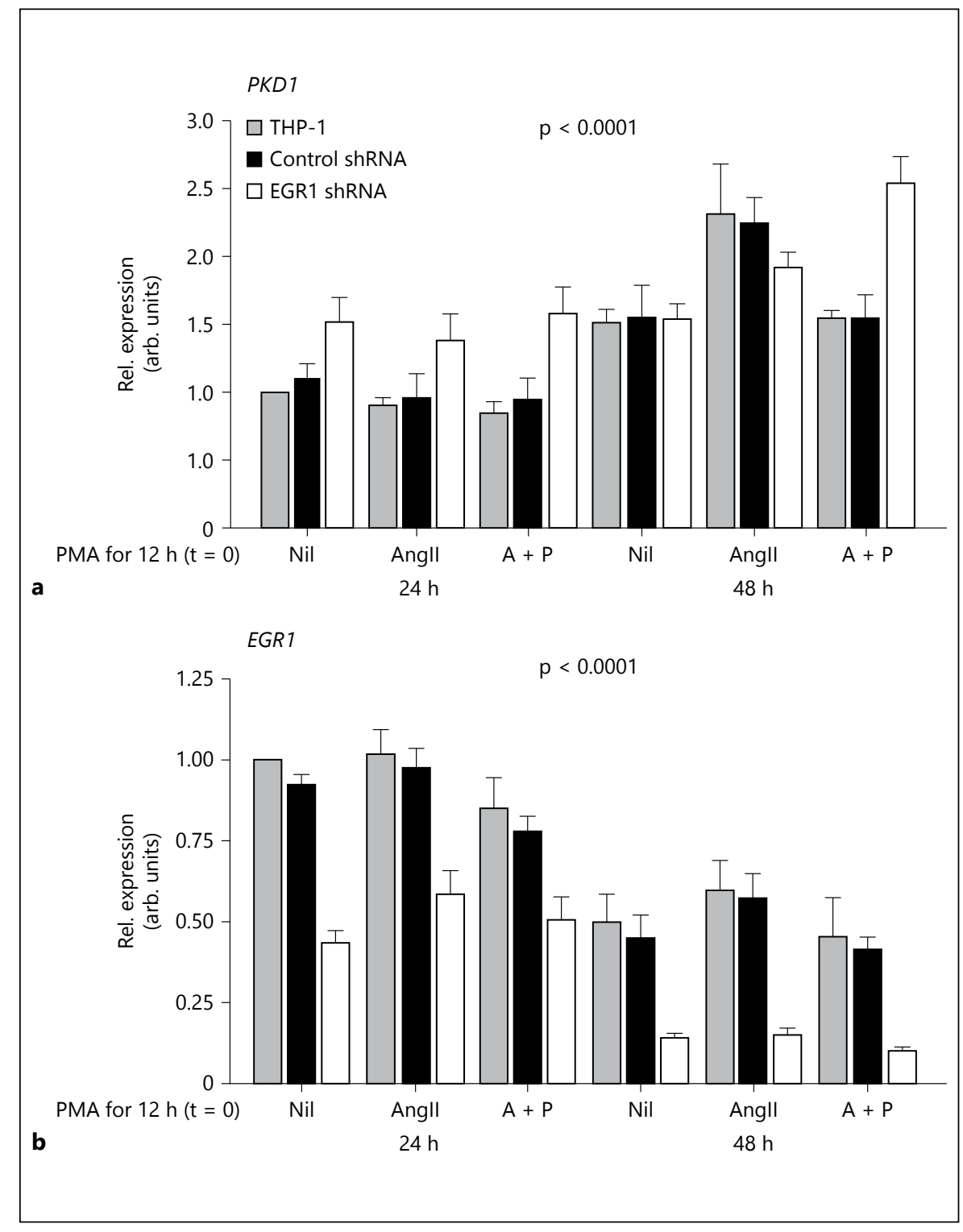

Table 1. Effect of Egr1 deficiency on changes in aortic diameter in $\mathrm{CaCl}_{2}$-induced aneurysm formation

\begin{tabular}{lll}
\hline & $\begin{array}{l}\text { Recipient Egrl } \\
\text { Donor Egr1 }\end{array}$ & $\begin{array}{l}\text { Recipient Egr1 } \\
\text { Donor Egr1 }\end{array}$ \\
\hline Animals, $\mathrm{n}$ & 14 & 15 \\
Pretreatment diameter, $\mu \mathrm{m}$ & $399.7 \pm 41.7$ & $452.8 \pm 50.7$ \\
Posttreatment diameter, $\mu \mathrm{m}$ & $564.9 \pm 139.1$ & $500.0 \pm 64.2^{*}$ \\
AAA development, \% & 50 & 0 \\
\% increase & $42.51 \pm 35.7$ & $10.43 \pm 19.2$ \\
Range of increase, $\mu \mathrm{m}$ & $0-235.2$ & $0-116.2$ \\
\hline \multirow{2}{*}{$* \mathrm{p}=0.048}$. & & \\
\hline
\end{tabular}

90

J Vasc Res 2015;52:81-93 DOI: $10.1159 / 000430986$
Conversely, experimentally induced atherosclerosis and inflammation is greatly diminished in EGR1 deficient mice [25].

The PKD1 gene encodes polycystin-1, a complex, membrane-spanning glycoprotein that contains a uniquely large extracellular N-terminal ectodomain with a $G$ protein-coupled receptor proteolytic site that can be cleaved from its transmembrane segment [27]. Intracellularly, polycystin-1 either interacts with the cytoplasmic tail of the PKD2 gene product, polycystin-2, or undergoes proteolytic cleavage to release a fragment from its $\mathrm{C}$ terminus, which translocates to the nucleus playing a role in gene transcription [28]. Mutations in the PKD1 gene are 
the cause of cyst formation in kidney, liver, pancreas and spleen, and they are linked to a high incidence of abdominal and intracranial aneurysms as well as to other cardiac defects [11-13, 29,30], suggesting a multifunctional/ multifactorial potential for polycystin-1. PKD1 is expressed in embryonic and adult kidney epithelial cells, but also in non-epithelial cells and in a variety of tissues and cells, from the vasculature (e.g. smooth muscle, endothelial cells) to hematopoietic cell lines [12, 30-32]. Consistent with our findings from THP-1 cells, the differentiation of K562 and HL60 cells to macrophages has been reported to coincide with the down-regulation of the PKD1 expression [32]. Similarly to THP-1 cells, K562 and HL60 cells also show an increase in EGR1 levels in response to PMA [33], suggesting an EGR1-mediated suppression of $P K D 1$ upon their differentiation to a macrophage lineage.

$P K D 1$ is tightly regulated; during early embryogenesis, it is highly expressed in many tissues and organs, but during adulthood it is maintained at low levels [34]. In ADPKD mouse models, $P K D 1$ mutations resulting in loss of heterozygocity, haploinsufficency or overexpression trigger renal and extrarenal phenotypes with a varying degree of penetrance, supporting a gene dosage-dependent mechanism $[12,13,30,35-37]$. $P K D 1^{-/-}$mice, apart from kidney cysts, present aortic dissections that closely resemble the dissections of the AngII AAA mouse model [37]. The vascular fragility and aneurysmal phenotypes of the ADPKD mouse models support the involvement of polycystin-1 in altered extracellular matrix production, integrity and maintenance $[12,37]$. In humans, ADPKD is also associated with aneurysms, which appear at a much greater incidence than in the rest of the population. The few studies that have assessed the frequency of aneurysms in the aorta report a higher prevalence from 1 to $10 \%$ in ADPKD patients [11,38-40]. However, down-regulation of $P K D 1$ in dissected human aortas has also been found independently of ADPKD in a gene expression profile analysis of patients that suffered acute aortic dissection of the ascending aorta, without having any other genetic background (e.g. Marfan's or Ehlers-Danlos syndrome) [14]. Similarly, whole genome expression profiling data showed $P K D 2$, a gene closely related to $P K D 1$, to be one of the top most down-regulated genes in human AAA [41].

EGR1 is an immediately induced transcription factor that has been shown to be involved in a variety of cellular responses from differentiation, growth and survival to growth arrest and apoptosis [21, 22]. In THP-1 cells, EGR1 expression is rapidly increased at the level of tran-

Role of EGR1 in Aortic Aneurysm scription, so there is an abrupt rise in the EGR1 mRNA and protein levels upon PMA stimulation. EGR1 binds to its consensus as a monomer and can be a direct/indirect activator of transcription depending on other interacting transcription factors, or a direct suppressor of transcription by displacement of a more positive factor (e.g. stimulating protein 1) [21,26]. Multiple lines of evidence have so far shown that EGR1 does not act as a simple on/off switch of transcription; its mechanism of action seems to depend on a concentration equilibrium of interacting factors and chromatin conformation changes that may change affinities among shared binding sites, allowing active displacement of one transcription factor by another $[21,23]$.

Our analysis of the $P K D 1$ proximal promoter revealed a putative EGR1 binding site, which we confirmed to be occupied and functional. A binding site for EGR factors (EGRFs) had been previously documented in the proximal promoter of $P K D 1$ in a study looking for conserved regulatory elements in multiple species [42]. The EGRF binding site was found to be conserved in mammals (i.e. humans, mice and dogs), but not in fish (i.e. Fugu rubripes), suggesting a functional role specific for EGRFs in mammal PKD1 regulation. Other conserved transcription factor binding sites that have been identified in the proximal $P K D 1$ promoter include multiple stimulating protein $1[42,43]$, E26 avian erythroblastosis virus transformation-specific sequence [40] and myeloid zinc finger 1 [42], the latter also pointing to a role for $P K D 1$ in hematopoietic lineages. Further, our comparative analyses of already publicly available EGR1 ChIP-seq data revealed a conserved EGR1 binding site within the PKD1 promoter and EGR1 peaks within the $P K D 2$ promoter. Since the expression of polycystin- 1 and polycystin- 2 is highly coordinated and the 2 proteins interact [34], elements common to the promoters of $P K D 1$ and $P K D 2$ are likely to be involved in a conserved regulatory mechanism. The fact that EGR1 shRNA resulted in an up-regulation of PKD1 expression in differentiating THP-1 monocytes confirms such a functional regulatory relationship for the first time and assigns it, largely, to the EGR1 member rather than to other EGRFs.

Inflammation plays a fundamental role in the initiation, growth and rupture of aneurysms [2, 4, 5, 41]. Extensive research over the past two decades has revealed mechanisms by which continued oxidative stress can lead to chronic inflammation. This chronic inflammation can, over time, compromise the homeostatic mechanisms able to redress these events. A drug treatment for aneurysm stabilisation has to be able to perturb oxidative 
stress-induced inflammation on the one hand and, since the disease is largely asymptomatic for most of its development, has to also be a drug of 'no harm'. EGR1 is an important regulating factor in many target genes induced during chronic inflammation, such as tissue factor, ICAM-1 and IL-1 $\beta$ [26]. Blocking either EGR1 or its downstream targets may present a new therapeutic opportunity for aneurysmal stabilisation. The use of antisense RNAs against EGR1 did show some benefit in lung transplantation [44]. In addition, IL1 $\beta$, a proven target of EGR1, as shown in the recently registered clinical trial (NCT02007252) exploring the therapeutic potential of IL1 $\beta$ antibodies on aneurysm growth, may support this mechanism.

\section{Acknowledgements}

The authors thank the staff of St George's Biological Research Facility for their exemplary skills in animal husbandry and compassionate attention to the needs of the animals in their care.

\section{Funding}

This work was supported by British Heart Foundation PG10/001/2008.

\section{Disclosure Statement}

The authors have no conflicts of interest to declare.

\section{References}

1 Sidloff D, Stather P, Dattani N, Bown M, Thompson J, et al: Aneurysm global epidemiology study: public health measures can further reduce abdominal aortic aneurysm mortality. Circulation 2014;129:747753.

2 Guo DC, Papke CL, He R, Milewicz DM: Pathogenesis of thoracic and abdominal aortic aneurysms. Ann N Y Acad Sci 2006;1085: 339-352.

$3 \mathrm{Lu} \mathrm{H}$, Rateri DL, Cassis LA, Daugherty A: The role of the renin-angiotensin system in aortic aneurysmal diseases. Curr Hypertens Rep 2008;10:99-106.

4 Curci JA, Liao S, Huffman MD, Shapiro SD, Thompson RW: Expression and localization of macrophage elastase (matrix metalloproteinase-12) in abdominal aortic aneurysms. J Clin Invest 1998;102:1900-1910.

5 Anidjar S, Dobrin PB, Eichorst M, Graham GP, Chejfec G: Correlation of inflammatory infiltrate with the enlargement of experimental aortic aneurysms. J Vasc Surg 1992;16: 139-147.

6 Golledge J, Cullen B, Rush C, Moran CS, Secomb E, et al: Peroxisome proliferator-activated receptor ligands reduce aortic dilatation in a mouse model of aortic aneurysm. Atherosclerosis 2010;210:51-56.

7 Jones A, Deb R, Torsney E, Howe F, Dunkley $\mathrm{M}$ : Rosiglitazone reduces the development and rupture of experimental aortic aneurysms. Circulation 2009;119:3125-3132.

8 Daugherty A, Cassis LA: Mouse models of abdominal aortic aneurysms. Arterioscler Thromb Vasc Biol 2004;24:429-434.

9 Spin JM, Hsu M, Azuma J, Tedesco MM, Deng A, et al: Transcriptional profiling and network analysis of the murine angiotensin II-induced abdominal aortic aneurysm. Physiol Genomics 2011;43:993-1003.

10 Nissen SE, Wolski K: Rosiglitazone revisited: an updated meta-analysis of risk for myocar- dial infarction and cardiovascular mortality. Arch Intern Med 2010;170:1191-1201.

11 Chapman JR, Hilson AJ: Polycystic kidneys and abdominal aortic aneurysms. Lancet 1980;1:646-647.

12 Kim K, Drummond I, IbraghimovBeskrovnaya $\mathrm{O}$, Klinger $\mathrm{K}$, Arnaout MA: Polycystin 1 is required for the structural integrity of blood vessels. Proc Natl Acad Sci U S A 2000;97:1731-1736.

13 Kurbegovic A, Cote O, Couillard M, Ward CJ, Harris PC, et al: Pkd1 transgenic mice: adult model of polycystic kidney disease with extrarenal and renal phenotypes. Hum Mol Genet 2010;19:1174-1189.

14 Müller BT, Modlich O, Prisack HB, Bojar H, Schipke JD, et al: Gene expression profiles in the acutely dissected human aorta. Eur J Vasc Endovasc Surg 2002;24:356-364.

15 Kubosaki A, Tomaru Y, Tagami M, Arner E, Miura $\mathrm{H}$, et al: Genome-wide investigation of in vivo EGR-1 binding sites in monocytic differentiation. Genome Biol 1999;10:R41.

16 Boyer LA, Lee TI, Cole MF, Johnstone SE, Levine SS: Core transcriptional regulatory circuitry in human embryonic stem cells. Cell 2005; 122:947-956.

17 Barrett T, Wilhite SE, Ledoux P, Evangelista C, Kim IF, et al: NCBI GEO: archive for functional genomics data sets - update. Nucleic Acids Res 2013;4:D991-D995.

18 Wang Y, Krishna S, Golledge J: The calcium chloride-induced rodent model of abdominal aortic aneurysm. Atherosclerosis 2013;226: 29-39.

19 Wilson WR, Anderton M, Choke EC, Dawson J, Loftus IM: Elevated plasma MMP1 and MMP9 are associated with abdominal aortic aneurysm rupture. Eur J Vasc Endovasc Surg 2008;35:580-584.

20 Varga T, Nagy L: Nuclear receptors, transcription factors linking lipid metabolism and immunity: the case of peroxisome prolifera- tor-activated receptor gamma. Eur J Clin Invest 2008;38:695-707.

21 Silverman ES, Collins T: Pathways of Egr-1-mediated gene transcription in vascular biology. Am J Pathol 1999;154:665-670.

22 Milbrandt J: A nerve growth factor-induced gene encodes a possible transcriptional regulatory factor. Science 1987;238:797-799.

23 Eyries M, Agrapart M, Alonso A, Soubrier F: Phorbol ester induction of angiotensin-converting enzyme transcription is mediated by Egr-1 and AP-1 in human endothelial cells via ERK1/2 pathway. Circ Res 2002;91:899-906.

24 Shin IS, Kim JM, Kim KL, Jang SY, Jeon ES, et al: Early growth response factor-1 is associated with intraluminal thrombus formation in human abdominal aortic aneurysm. J Am Coll Cardiol 2009;53:792-799.

25 Harja E, Bucciarelli LG, Lu Y, Stern DM, Zou YS, et al: Early growth response-1 promotes atherogenesis: mice deficient in early growth response- 1 and apolipoprotein E display decreased atherosclerosis and vascular inflammation. Circ Res 2004;94:333-339.

26 Khachigian LM: Early growth response- 1 in cardiovascular pathobiology. Circ Res 2006; 98:186-191.

27 Ponting CP, Hofmann K, Bork P: A latrophilin/CL-1-like GPS domain in polycystin-1. Curr Biol 1999;9:R585-R588.

28 Chauvet V, Tian X, Husson H, Grimm DH, Wang $T$, et al: Mechanical stimuli induce cleavage and nuclear translocation of the polycystin-1 C terminus. J Clin Invest 2004; 114:1433-1443.

29 Drummond IA: Polycystins, focal adhesions and extracellular matrix interactions. Biochim Biophys Acta 2011;1812:1322-1326.

30 Boulter C, Mulroy S, Webb S, Fleming S, Brindle K, et al: Cardiovascular, skeletal, and renal defects in mice with a targeted disruption of the Pkd1 gene. Proc Natl Acad Sci U S A 2001; 98:12174-12179. 
31 Ibraghimov-Beskrovnaya O, Dackowski WR, Foggensteiner L, Coleman N, Thiru S, et al: Polycystin: in vitro synthesis, in vivo tissue expression, and subcellular localization identifies a large membrane-associated protein. Proc Natl Acad Sci U S A 1997;94:63976402.

32 Aguiari G, Piva R, Manzati E, Mazzoni E, Augello G, et al: K562 erythroid and HL60 macrophage differentiation downregulates polycystin, a large membrane-associated protein. Exp Cell Res 1998;244:259-267.

33 Danilenko M, Wang X, Studzinski GP: Carnosic acid and promotion of monocytic differentiation of HL60-G cells initiated by other agents. J Natl Cancer Inst 2001;93:12241233.

34 Chauvet V, Qian F, Boute N, Cai Y, Phakdeekitacharoen B, Onuchic LF, Attie-Bitach T, Guicharnaud L, Devuyst O, Germino GG, Gubler MC: Expression of PKD1 and PKD2 transcripts and proteins in human embryo and during normal kidney development. Am J Pathol 2002;160:973-983.
35 Thivierge C, Kurbegovic A, Couillard M, Guillaume R, Cote $\mathrm{O}$, et al: Overexpression of PKD1 causes polycystic kidney disease. Mol Cell Biol 2006;26:1538-1548.

36 Muto S, Aiba A, Saito Y, Nakao K, Nakamura $\mathrm{K}$, et al: Pioglitazone improves the phenotype and molecular defects of a targeted Pkd1 mutant. Hum Mol Genet 2002;11:1731-1742.

37 Hassane S, Claij N, Lantinga-van Leeuwen IS, Van Munsteren JC, Van LN, Hanemaaijer R, Breuning $\mathrm{MH}$, et al: Pathogenic sequence for dissecting aneurysm formation in a hypomorphic polycystic kidney disease 1 mouse model. Arterioscler Thromb Vasc Biol 2007; 27:2177-2183.

38 Bailey MA, Griffin KJ, Windle AL, Lines SW, Scott DJ: Cysts and swellings: a systematic review of the association between polycystic kidney disease and abdominal aortic aneurysm. Ann Vasc Surg 2013;27:123-128.

39 Roodvoets AP: Aortic aneurysms in presence of kidney disease. Lancet 1980;1:14131414.
40 Torra R, Nicolau C, Badenas C, Bru C, Perez $\mathrm{L}$, et al: Abdominal aortic aneurysms and autosomal dominant polycystic kidney disease. J Am Soc Nephrol 1996;7:2483-2486.

41 Lenk GM, Tromp G, Weinsheimer S, Gatalica $Z$, Berguer R, et al: Whole genome expression profiling reveals a significant role for the immune function in human abdominal aortic aneurysms. BMC Genomics 2007;8:237.

42 Lantinga-van Leeuwen IS, Leonhard WN, Dauwerse H, Baelde HJ, van Oost BA, et al: Common regulatory elements in the polycystic kidney disease 1 and 2 promoter regions. Eur J Hum Genet 2005;13:649-659.

43 Islam MR, Puri S, Rodova M, Magenheimer BS, Maser RL, et al: Retinoic acid-dependent activation of the polycystic kidney disease- 1 (PKD1) promoter. Am J Physiol Renal Physiol 2008;295:F1845-F1854.

44 Okada M, Fujita T, Sakaguchi T, Olson KE, Collins T, Stern DM, et al: Extinguishing Egr1-dependent inflammatory and thrombotic cascades following lung transplantation. FASEB J 2001;15:2757-2759. 